\title{
Phase retrieval between overlapping orders in coherent Fourier scatterometry using scanning
}

\author{
N. Kumar \\ N.Nitishkumar@tudelft.nl \\ O. El Gawhary
}

\section{S. Roy}

\section{S. F. Pereira}

H. P. Urbach
Optics Research Group, Department of Imaging Science and Technology, Faculty of Applied Sciences, Delft University of Technology, P. O. Box 5046, 2600GA Delft, The Netherlands

Optics Research Group, Department of Imaging Science and Technology, Faculty of Applied Sciences, Delft University of Technology, P. O. Box 5046, 2600GA Delft, The Netherlands VSL Dutch Metrology Institute, Thijsseweg 11, 2600 AR Delft, The Netherlands

Optics Research Group, Department of Imaging Science and Technology, Faculty of Applied Sciences, Delft University of Technology, P. O. Box 5046, 2600GA Delft, The Netherlands

Optics Research Group, Department of Imaging Science and Technology, Faculty of Applied Sciences, Delft University of Technology, P. O. Box 5046, 2600GA Delft, The Netherlands

Optics Research Group, Department of Imaging Science and Technology, Faculty of Applied Sciences, Delft University of Technology,P. O. Box 5046, 2600GA Delft, The Netherlands

Non-interferometric phase retrieval from the intensity measurements in Coherent Fourier Scatterometry (CFS) is presented using a scanning focused spot. Formulae to determine the state of polarization of the scattered light and to retrieve the phase difference between overlapping scattered orders are given. The scattered far field is rigorously computed and the functionality of the method is proved with experimental results. [DOI: http://dx.doi.org/10.2971/jeos.2013.13048]

Keywords: Optical metrology, scatterometry, RCWA, coherent Fourier scatterometry, grating, phase retrieval, temporal phase shifting interferometry

\section{INTRODUCTION}

In conventional incoherent optical scatterometry (IOS), light from an incoherent source interacts with the scatterer and the diffracted far field intensity map is recorded [1, 2]. The energy distribution in the diffracted far field depends on the material composition and the geometrical properties of the scatterer that one wants to reconstruct. In the lithography industry, smallest details of the scatterer (grating) are smaller than the wavelength used for inspection. Therefore imaging the structure is not an option. Forward modeling in scatterometry can be rigorously solved. The inverse problems in scatterometry belongs to the family of ill-posed problems in electromagnetism, but by using a-priori information about the scatterer, reconstruction of the grating is possible to a certain extent. IOS is an established method of lithographic process inspection [3]-[5], but with continuing shrinkage of the features written by lithographic machines, a more sensitive, repeatable and robust method for semiconductor metrology is desirable.

Coherent Fourier Scatterometry (CFS) is very useful in applications where there are overlapping orders present in the far field. The applications include overlay metrology, target alignment etc. In the last few years, we have investigated and implemented CFS, using a focused spot from a coherent source of light as illumination [6]-[8]. This focused spot is scanned over the grating such that there is an overlap between the consecutive scan positions. In contrast to IOS, where only the field intensities are accessible and there is no phase informa- tion present, in CFS it is possible to retrieve phase information from the intensity measurements. There are techniques available to transform the intensity information into phase such as interferometry [9]-[12], but they are either technically demanding or time consuming for the real time implementation in the semiconductor industry.

In this paper, we show how the phase difference between overlapping diffracted orders of the grating for two orthogonal states of polarization can be retrieved. The setup resembles a common path interferometer and therefore the phase retrieval by our method is robust [13]. In our case, the zeroth order diffracted beam acts as the reference beam and the higher diffracted order acts as the object beam. Phase shift between the zeroth and the higher diffracted order is achieved by translating the grating. We derive an analytic phase relationship between the diffracted orders of the reflected far field in terms of intensities acquired for different scan positions within a single period of the grating. The situation is similar to Temporal Phase Shifting Interferometry (TPSI) [14, 15]. TPSI requires capturing multiple interference patterns, generated at different times by shifting the phase of one of the interfering beams by a predetermined phase shift. In our case, the zeroth order diffracted beam acts as the reference beam and the phase shift between the zeroth and the higher diffracted order is achieved by translating the grating. 
The content of the paper is as follows: In Section 2, we present the mathematical formulation of the method. In Section 3, the experimental setup is described and experimental and simulations results are given. Section 4 contains the conclusions.

\section{PHASE ANALYSIS}

In Figure 1(a), a periodic grating illuminated by a focused light beam is shown. The shape of the grating is defined by the period, midCD, height and side wall angles (SWA) as shown in Figure 1(b). An additional quantity called 'bias' is defined as the position of the grating relative to the optical axis of the focusing system. The bias $=0$ position can be chosen arbitrary, but usually we define it as the position when the optical axis bi-sects the MidCD as indicated by the dotted vertical line in Figure 1(b). Throughout the paper, we use a right-handed coordinate system $x y z$ as shown in Figure $1(\mathrm{a}($. The $x$-axis and $y$-axis are parallel and perpendicular to the spatial frequency vector $\vec{g}$ of the grating, respectively. The $z$-axis is parallel to the optical axis of the focusing system with $z$ increasing parallel to the direction of the incident field. A positive or negative bias value means that the grating is displaced parallel to the $x$-axis over a distance specified by the bias value. MidCD, height, SWA and bias are collectively the grating parameters that we want to retrieve from the reflected data (see Figure 1(b)). In
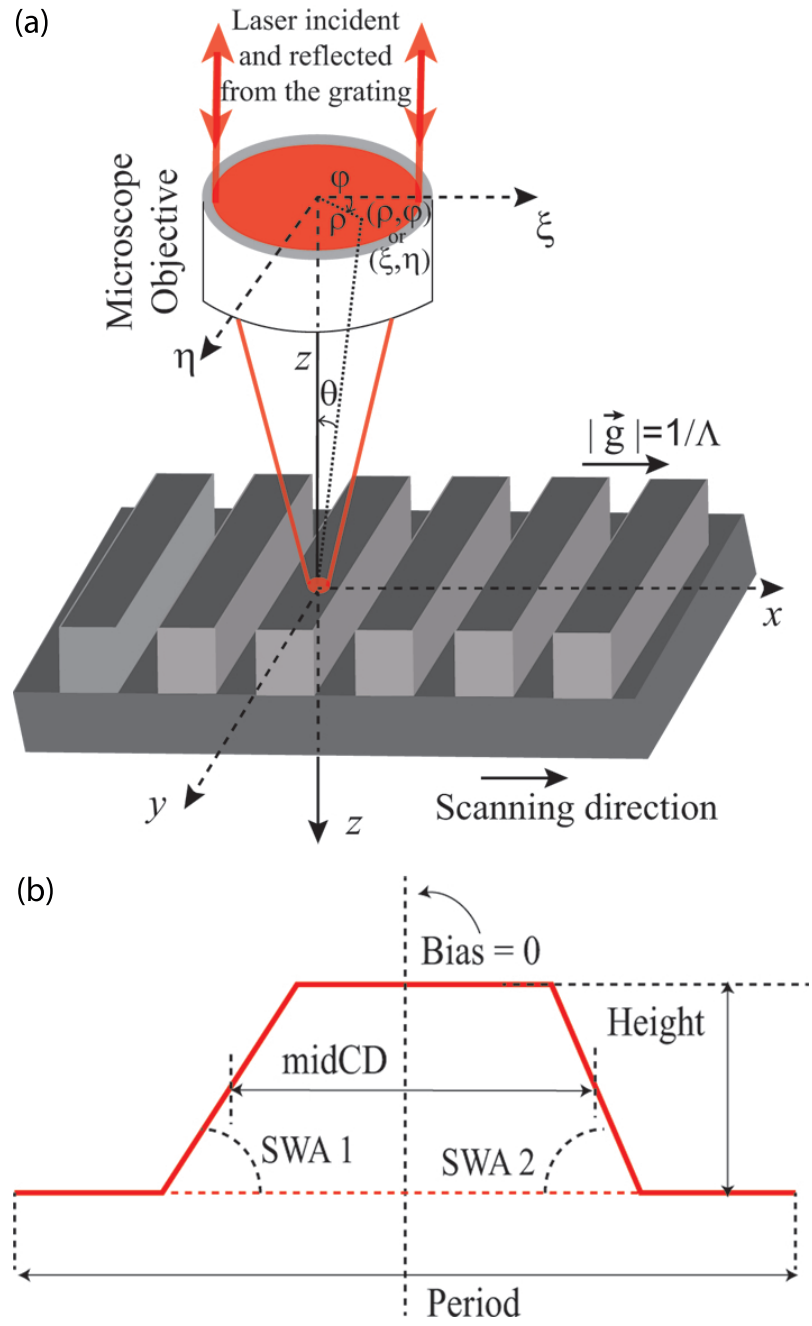

FIG. 1 (a) Scheme of the grating illumination. (b) Cross-section in the ( $x-z)$ plane of one period of the grating, showing the grating parameters. addition to the $(x, y, z)$ system, we introduce the coordinate system $(\xi, \eta)$ in the pupil of the focusing lens, where $\xi$ is parallel to the $x$ and $\eta$ is parallel to $y$.

Let $\left(\xi^{i}, \eta^{i}\right)$ be a point in the entrance pupil of the lens and let $\vec{E}_{\text {lens }}^{i}\left(\xi^{i}, \eta^{i}\right)$ be the real electric field in the entrance pupil that is focused by the lens :

$$
\begin{aligned}
& \vec{E}_{\text {lens }}^{i}\left(\xi^{i}, \eta^{i}\right)=\Re\left[\vec{a}^{i}\left(\xi^{i}, \eta^{i}\right) \exp (-i \omega t)\right]= \\
& \quad \Re\left\{\left[a_{\xi}^{i}\left(\xi^{i}, \eta^{i}\right) \hat{\xi}+a_{\eta}^{i}\left(\xi^{i}, \eta^{i}\right) \hat{\eta}\right] \exp (-i \omega t)\right\},
\end{aligned}
$$

where $\vec{a}^{i}\left(\xi^{i}, \eta^{i}\right)$ is the complex incident field in the entrance pupil. The field in Eq. (1) is written as the sum of fields that are linearly polarized parallel to the $\hat{\xi}$ and $\hat{\eta}$ directions. We can also write the incident field $\vec{a}^{i}\left(\xi^{i}, \eta^{i}\right)$ as the sum of radially and azimuthally polarized fields:

$$
\vec{a}^{i}\left(\xi^{i}, \eta^{i}\right)=a_{\rho}^{i}\left(\xi^{i}, \eta^{i}\right) \hat{\rho}+a_{\varphi}^{i}\left(\xi^{i}, \eta^{i}\right) \hat{\varphi} .
$$

where, $\rho$ and $\varphi$ are polar coordinates and

$$
\begin{aligned}
& \hat{\rho}=\cos \varphi \hat{\xi}+\sin \varphi \hat{\eta}, \\
& \hat{\varphi}=-\sin \varphi \hat{\xi}+\cos \varphi \hat{\eta}, \\
& \xi^{i}=\rho_{i} \cos \varphi^{i}, \eta^{i}=\rho_{i} \sin \varphi^{i} .
\end{aligned}
$$

The relation between the amplitude in the cylindrical and cartesian coordinates is given by

$$
\begin{aligned}
& \left(\begin{array}{c}
a_{\xi}^{i} \\
a_{\eta}^{i}
\end{array}\right)=\left(\begin{array}{cc}
\cos \varphi^{i} & -\sin \varphi^{i} \\
\sin \varphi^{i} & \cos \varphi^{i}
\end{array}\right)\left(\begin{array}{c}
a_{\rho}^{i} \\
a_{\varphi}^{i}
\end{array}\right) \equiv \\
& \underline{\underline{\Omega}}\left(\varphi_{i}\right)\left(\begin{array}{c}
a_{\rho}^{i} \\
a_{\varphi}^{i}
\end{array}\right) \text {. }
\end{aligned}
$$

To a given point $\left(\xi^{i}, \eta^{i}\right)$ in the entrance pupil of the lens, there corresponds an incident plane wave with wavevector $\vec{k}^{i}$ in the angular spectrum of the focused spot, which is given by

$$
\vec{k}^{i}=k_{x}^{i} \hat{x}+k_{y}^{i} \hat{y}+k_{z}^{i} \hat{z},
$$

with:

$$
\begin{aligned}
& k_{x}^{i}=-k_{0} n \frac{\xi^{i}}{f}, \\
& k_{y}^{i}=-k_{0} n \frac{\eta^{i}}{f}, \\
& k_{z}^{i}=\sqrt{k_{0}^{2} n^{2}-k_{x}^{i^{2}}-k_{y}^{i^{2}}},
\end{aligned}
$$

where $k_{0}$ is the wavenumber in vacuum, $f$ is the focal length of the focusing lens and $n$ is the refractive index of the medium between lens and the grating (in our case $n=1$ ). Assuming an aplanatic lens, it follows from the theory of Ignatowski [16] and Richards \& Wolf [17] that the radial and azimuthal components in the entrance pupil are transferred by the lens into $\mathbf{S}$ and $\mathbf{P}$ polarized electric field vectors, respectively. In fact, let $(\theta, \varphi)$ be spherical coordinates, such that

$$
\begin{aligned}
& k_{x}^{i}=k_{0} n \cos \varphi^{i} \sin \theta^{i}, \\
& k_{y}^{i}=k_{0} n \sin \varphi^{i} \sin \theta^{i}, \\
& k_{z}^{i}=k_{0} n \cos \theta^{i} .
\end{aligned}
$$


Then, the electric field of the plane wave $\hat{k}^{i}$ is

$$
\vec{E}^{i}\left(k_{x}^{i}, k_{y}^{i}\right)=\sqrt{\cos \theta^{i}}\left[a_{\rho}^{i}\left(\xi^{i}, \eta^{i}\right) \hat{\theta}+a_{\varphi}^{i}\left(\xi^{i}, \eta^{i}\right) \hat{\varphi}\right] .
$$

The factor $\sqrt{\cos \theta^{i}}$ is due to the conservation of energy flux [18].

In the remainder of this paper, we assume that the state of polarization, the amplitude and the phase of the incident field have been determined. This means that $\vec{a}_{\tilde{\xi}}^{i}\left(\xi^{i}, \eta^{i}\right)$ and $\vec{a}_{\eta}^{i}\left(\xi^{i}, \eta^{i}\right)$ are known complex functions of position $\left(\xi^{i}, \eta^{i}\right)$ in the pupil (except for an overall constant phase). The incident plane wave is partially reflected by the grating giving rise to the reflected propagating orders with wave vectors

$$
\begin{gathered}
\vec{k}^{s, m}=k_{x}^{s, m} \hat{x}+k_{y}^{s, m} \hat{y}-k_{z}^{s, m} \hat{z}= \\
\left(k_{x}^{i}+\frac{2 \pi m}{\Lambda}\right) \hat{x}+k_{y}^{i} \hat{y}-k_{z}^{s, m} \hat{z} .
\end{gathered}
$$

Where the superscript $s$ indicates the scattered direction, $\Lambda$ is the pitch of the grating, $m$ is an integer representing the diffracted order such that

$$
\left(k_{x}^{i}+\frac{2 \pi m}{\Lambda}\right)^{2}+k_{y}^{i^{2}} \leq\left(k_{0} n\right)^{2}
$$

and $k_{z}^{s, m}=\sqrt{k_{0}^{2} n^{2}-\left(k_{x}^{i}+\frac{2 \pi m}{\Lambda}\right)^{2}-\left(k_{y}^{i}\right)^{2}}$. The electric field of the $m^{\text {th }}$ diffracted order is written as

$$
\vec{E}^{s, m}\left(k_{x}^{s, m}, k_{y}^{s, m}\right)=\underline{r}_{m}\left(k_{x}^{i}, k_{y}^{i}\right) \vec{E}^{i}\left(k_{x}^{i}, k_{y}^{i}\right) .
$$

Where, ${ }_{=}^{r}\left(k_{x}^{i}, k_{y}^{i}\right)$ is a $(2 \times 2)$ matrix on the $(\hat{\theta}, \hat{\varphi})$ bases that relates the incident and the $m^{\text {th }}$ reflected wave vector. We can also write

$$
\begin{aligned}
& k_{x}^{s, m}=k_{0} n \cos \varphi^{s, m} \sin \theta^{s, m}, \\
& k_{y}^{s, m}=k_{0} n \sin \varphi^{s, m} \sin \theta^{s, m}, \\
& k_{z}^{s, m}=-k_{0} n \cos \theta^{s, m} .
\end{aligned}
$$

The information about the grating is contained in the matrices $\underline{r}_{m}\left(k_{x}^{i}, k_{y}^{i}\right)$, and hence it is important to retrieve as much information as possible (amplitude and phase) from these matrices to reconstruct the grating parameters. The reflected orders are collected back by the same focusing objective and the far field is captured by the CCD. By inverting Eq. (5), we obtain the electric field $\vec{a}^{s, m}$ of the $m^{\text {th }}$ reflected order in the exit pupil of the collimator:

$$
\begin{aligned}
& \vec{a}^{s, m}\left(\xi^{s, m}, \eta^{s, m}\right)= \\
& \frac{1}{\sqrt{\cos \theta^{s, m}}}\left[\vec{E}_{\theta}^{s, m}\left(k_{x}^{s, m}, k_{y}^{s, m}\right) \hat{\rho}+\vec{E}_{\varphi}^{s, m}\left(k_{x}^{s, m}, k_{y}^{s, m}\right) \hat{\varphi}\right] .
\end{aligned}
$$

Where

$$
\xi^{s, m}=-\frac{k_{x}^{s, m}}{k_{0} n} f, \quad \eta^{s, m}=-\frac{k_{y}^{s, m}}{k_{0} n} f
$$

Using Eq. (5), (8) and (9) we find that the $(\xi, \eta)$-components of the reflected and the incident fields in the pupil are related as follows:

$$
\begin{aligned}
& \left(\begin{array}{c}
a_{\tilde{\xi}}^{s, m}\left(\xi^{s, m}, \eta^{s, m}\right) \\
a_{\eta}^{s, m}\left(\xi^{s, m}, \eta^{s, m}\right)
\end{array}\right)= \\
& \sqrt{\frac{\cos \theta^{i}}{\cos \theta^{s, m}} \Omega\left(\varphi^{s, m}\right) \underline{r}_{m}\left(k_{x}^{i}, k_{y}^{i}\right) \underline{\underline{\Omega}}\left(\varphi^{i}\right)^{-1}\left(\begin{array}{c}
a_{\tilde{\zeta}}^{i}\left(\xi^{i}, \eta^{i}\right) \\
a_{\eta}^{i}\left(\xi^{i}, \eta^{i}\right)
\end{array}\right)=} \\
& \stackrel{\widetilde{r}}{=}_{m}\left(k_{x}^{i}, k_{y}^{i}\right)\left(\begin{array}{c}
a_{\tilde{\xi}}^{i}\left(\xi^{i}, \eta^{i}\right) \\
a_{\eta}^{i}\left(\xi^{i}, \eta^{i}\right)
\end{array}\right) .
\end{aligned}
$$

Where

$$
\begin{aligned}
& \stackrel{\widetilde{r}_{m}}{=}\left(k_{x}^{i}, k_{y}^{i}\right)= \\
& \quad \sqrt{\frac{\cos \theta^{i}}{\cos \Omega^{s, m}} \underline{\underline{ }}\left(\varphi^{s, m}\right) \underline{\underline{r}}_{m}\left(k_{x}^{i}, k_{y}^{i}\right) \underline{\underline{\Omega}}\left(\varphi^{i}\right)^{-1} .}
\end{aligned}
$$

Here $\widetilde{\widetilde{r}}_{m}\left(k_{x}^{i}, k_{y}^{i}\right)$ is the reflection matrix that relates the field of the $m^{t h}$ reflected order in the pupil of the objective to the incident field in the pupil.

Let us now look at a fixed scattering direction given by the wave vector $\vec{k}^{s}=k_{x}^{s} \hat{x}+k_{y}^{s} \hat{y}-k_{z}^{s} \hat{z}$. The corresponding point $\left(\xi^{\mathcal{S}}, \eta^{\mathcal{S}}\right)$ in the pupil is

$$
\xi^{s}=\frac{-k_{x}^{s}}{k_{0} n} f, \quad \eta^{s}=\frac{-k_{y}^{s}}{k_{0} n} f .
$$

It has been shown that in the reflected field, when there is no overlap between diffracted orders, CFS and IOS are equivalent [6]. When there is an overlap in the diffracted orders, CFS gives better sensitivity and more accurate reconstruction of the grating parameters provided scanning is applied [6, 7]. The number of overlapping orders is determined by the value of the so called overlap parameter:

$$
F=\frac{\lambda}{\Lambda \cdot N A}
$$

The smaller the value of $F$, the more orders overlap. In particular, when $1 \leq F \leq 2$ then only the zeroth and first orders overlap in part of the lens pupil while the first and minus first diffracted orders do not overlap and all higher diffracted orders are either evanescent or not captured by the lens. For simplicity, in the remainder of this paper we assume that $1 \leq F \leq 2$ but, the analysis can be extended to other values of $F$ when more orders overlap.

\subsection{Pupil points with no overlapping orders}

For a particular point $\left(\xi^{S}, \eta^{S}\right)$ in a region without overlapping orders, only the specular reflected wave contributes to the measured intensity. Hence the total field is given by

$$
\left(\begin{array}{c}
a_{\tilde{\xi}}^{s, 0}\left(\xi^{s}, \eta^{s}\right) \\
a_{\eta}^{s, 0}\left(\xi^{s}, \eta^{s}\right)
\end{array}\right)=\widetilde{\widetilde{r}}_{=0}\left(k_{x}^{i}, k_{y}^{i}\right)\left(\begin{array}{c}
a_{\tilde{\xi}}^{i}\left(\xi^{i}, \eta^{i}\right) \\
a_{\eta}^{i}\left(\xi^{i}, \eta^{i}\right)
\end{array}\right) .
$$

We write the complex matrix $\underset{=}{\widetilde{r}_{0}}$ on the $(\xi, \eta)$ basis as

$$
\underline{\underline{r}}_{0}=\left(\begin{array}{ll}
\widetilde{\widetilde{r}}_{0, \xi \xi} & \widetilde{\underline{r}}_{0, \eta \xi} \\
\widetilde{\widetilde{r}}_{0, \xi \eta} & \underline{\widetilde{r}}_{0, \eta \eta}
\end{array}\right) \text {. }
$$


The subscripts $\xi \xi, \xi \eta, \eta \xi$ and $\eta \eta$ correspond to the set of input and output states of polarization. In order to retrieve the amplitudes of all four elements of the matrix $\widetilde{\underline{r}}_{0}$ and their phase differences, two polarizers are used in the setup. In this way the complex specular reflection matrix in the direction of a pupil point in a region without overlap can be completely retrieved except for an ambiguous common constant phase. We explain the procedure here briefly.

First, we use a linear polarizer in the incident beam just before the pupil and a linear polarizer in the reflected beam at the detector. By setting these polarizers alternatively parallel to the $\xi$ and $\eta$ axis and measuring the intensity, the moduli $\left|\underline{\underline{r}}_{0, \mu, v}\right|$, $(\mu, v=\xi, \eta)$ of all four elements of the reflection matrix are found.

Then the phase difference between the elements of the reflection matrix are determined in the following way: we choose the polarizer in the incident beam parallel to the $\xi$ axis and $\eta$ axis alternatively and the polarizer in the reflected beam at $45^{\circ}$ to the $\xi$ and $\eta$ axis and measure the intensity. From this, the

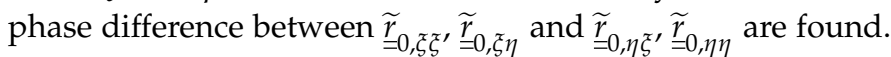
Then we put the input polarizer at $45^{\circ}$ w.r.t $\xi$ axis and the reflected intensity is measured with the polarizer parallel to $\xi$ and $\eta$ axis respectively at the detector. From this measure-

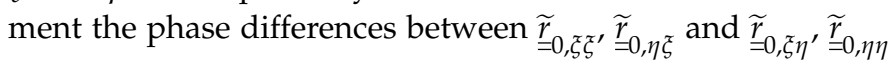
are found. In this way, the complex reflection matrix ${\underset{\widetilde{r}}{=}}_{0}$ is determined except for an overall constant phase.

\subsection{Pupil points where reflected orders overlap}

Let $\left(\xi^{S}, \eta^{S}\right)$ now be a point of the lens pupil where two reflected orders overlap (since we have assumed that $1<F<2$, no more than two orders overlap). To be specific, let $\left(\xi^{\mathcal{S}}, \eta^{\mathcal{S}}\right)$ be in the region of overlap of the $0^{\text {th }}$ reflected order (i.e. the specular reflected wave) and the $1^{\text {st }}$ reflected order. In this case, there is an incident wave with wave vector $\vec{k}^{i, 0}=$ $k_{x}^{i, 0} \hat{x}+k_{y}^{i, 0} \hat{y}+k_{z}^{i, 0} \hat{z}$, such that $\left|\vec{k}^{i, 0}\right|=\left|\vec{k}^{s}\right|$ and the corresponding pupil point $\left(\xi^{i, 0}, \eta^{i, 0}\right)=\left(-\xi^{\mathcal{S}},-\eta^{\mathcal{S}}\right)$, of which the specular reflected order is in the direction of $\vec{k}^{s}$. Furthermore there is an incident wave vector $\vec{k}^{i, 1}=k_{x}^{i, 1} \hat{x}+k_{y}^{i, 1} \hat{y}+k_{z}^{i, 1} \hat{z}$ with corresponding pupil point $\left(\xi^{i, 1}, \eta^{i, 1}\right)=\left(-\frac{f}{k_{0} n} k_{x}^{i, 1},-\frac{f}{k_{0} n} k_{y}^{i, 1}\right)$ of which the $1^{\text {st }}$ reflected order is in the direction of $\vec{k}^{s}$. There holds:

$$
\vec{k}^{i, 1}=\vec{k}^{s}+\frac{2 \pi}{\Lambda} \hat{x}
$$

The total reflected electric field in point $\left(\xi^{\mathcal{S}}, \eta^{\mathcal{S}}\right)$ of the exit pupil is the coherent sum of the field of the zeroth reflected order of incident wave vector $\vec{k}^{i, 0}$ and the $1^{\text {st }}$ reflected order of the incident wave vector $\vec{k}^{i, 1}$. Hence :

$$
\begin{gathered}
\vec{a}^{s}\left(\xi^{s}, \eta^{s}\right)=\underline{\widetilde{r}}_{0}\left(\xi^{i, 0}, \eta^{i, 0}\right) \vec{a}^{i}\left(\xi^{i, 0}, \eta^{i, 0}\right) \\
+\underline{\underline{r}}_{=}\left(\xi^{i, 1}, \eta^{i, 1}\right) \vec{a}^{i}\left(\xi^{i, 1}, \eta^{i, 1}\right) .
\end{gathered}
$$

This relation is valid for zero bias. When the grating is translated over the distance $\Delta x$ with respect to the zero bias position, the phases of the reflection matrices change and then $\widetilde{r}_{1}$ gets an additional phase of

$$
\Delta \Phi=\frac{2 \pi \Delta x}{\Lambda}
$$

with respect to $\underline{\underline{r}}_{0}$. The total reflected field in point $\left(\xi^{S}, \eta^{\mathcal{S}}\right)$ is then

$$
\begin{aligned}
& \vec{a}^{s, \Delta x}\left(\xi^{\mathcal{S}}, \eta^{s}\right)=\underline{\widetilde{r}}_{=0}\left(\xi^{i, 0}, \eta^{i, 0}\right) \vec{a}^{i}\left(\xi^{i, 0}, \eta^{i, 0}\right) \\
& +\underline{\widetilde{r}}_{1}\left(\xi^{i, 1}, \eta^{i, 1}\right) \vec{a}^{i}\left(\xi^{i, 1}, \eta^{i, 1}\right) \exp \left(\frac{i 2 \pi \Delta x}{\Lambda}\right) .
\end{aligned}
$$

We use again two polarizers in the setup: one in the incident beam immediately before the entrance pupil and the other in the reflected beam. First, suppose that both polarizers are parallel to $\xi$-direction. The measured reflected intensity for bias $\Delta x$ is given by

$$
\begin{aligned}
& I_{\xi \xi}^{\Delta x}\left(\xi^{s}, \eta^{s}\right)=\left|\vec{a}^{s, \Delta x}\left(\xi^{s}, \eta^{s}\right)\right|^{2}=\left|\underline{\underline{r}}_{0, \xi \xi}\left(\xi^{i, 0}, \eta^{i, 0}\right) \vec{a}_{\xi}^{i}\left(\xi^{i, 0}, \eta^{i, 0}\right)\right|^{2} \\
& +||_{=1, \xi \xi}\left(\xi^{i, 1},\left.\eta^{i, 1} \vec{a}_{\xi}^{i}\left(\xi^{i, 1}, \eta^{i, 1}\right)\right|^{2}\right. \\
& +2 \Re\left[\widetilde{\underline{r}}_{=0, \xi \xi}\left(\xi^{i, 0}, \eta^{i, 0}\right) \vec{a}_{\xi}^{i}\left(\xi^{i, 0}, \eta^{i, 0}\right) \cdot\right. \\
& \left.\quad \widetilde{\widetilde{r}}_{1, \xi \xi}\left(\xi^{i, 1}, \eta^{i, 1}\right)^{*} \vec{a}_{\xi}^{i}\left(\xi^{i, 1}, \eta^{i, 1}\right)^{*} \exp \left(\frac{-i 2 \pi \Delta x}{\Lambda}\right)\right]
\end{aligned}
$$

where the subscript $\xi \xi$ correponds to $\xi$ input and $\xi$ output polarization respectively. Because the incident field is assumed to be known, the unknown absolute values $\left|\underline{\underline{r}}_{0, \xi \xi}\right|,\left|\underline{\underline{r}}_{1, \xi \xi}\right|$ and the phase difference of ${\underset{=}{\tilde{r}}}_{0, \xi \xi}$ and $\underline{\underline{\underline{r}}}_{1, \xi \xi}$ can be determined from measuring $I_{\tilde{\zeta} \xi}^{\Delta x}$ for (at least) three scan positions $\Delta x$. By setting the linear polarizers alternatively parallel to the $\xi$ - and $\eta$-axis we obtain the moduli and phase differences of all elements of the two reflection matrices in the same row and column, i.e., of all $\underline{\underline{r}}_{0, \mu \nu}$ and ${\underset{\underline{r}}{\underline{r}}}_{1, \mu \nu}$ for $\mu, v=\xi, \eta$.

Note that when $\Delta x$ is such that the corresponding phase change $\Delta \Phi$ is in steps of $\pi / 2$, we can implement the bucket algorithm for phase retrieval [15]. The $0^{\text {th }}$ and $1^{\text {st }}$ diffracted orders act as the reference and the object beam respectively in the shear interferometer. The phase retrieval is thus very similar to TPSI, where one of the interfering beam is shifted by a phase difference of $\pi / 2$ (Appendix A).

Generalizing the proceeding scanning method to the case of more than two overlapping orders is straightforward. Due to a translation over $\Delta x$, the reflection matrix of the $m^{\text {th }}$ reflected order obtains a relative phase change with respect to the reflection matrix of the specular (i.e. $0^{\text {th }}$ ) order matrix given by:

$$
\Delta \Phi_{m}=\frac{2 \pi m \Delta x}{\Lambda} .
$$

When there are in total $M$ overlapping orders contributing to the total reflected field in a particular point of the lens pupil, we need at least $(2 M+1)$ scan positions to retrieve all amplitudes and phase differences of the reflection matrices.

By the described scanning procedure we have retrieved the absolute values of all elements in the same row and column of the contributing reflection matrices ${\underset{\underline{r}}{\underline{r}}}_{0}$ and ${\underset{\underline{r}}{=}}_{1}$ and the phase differences between them. Next, we want to determine the phase differences between elements in different rows and 
columns. This is done as described previously. By setting the polarizer in the incident beam parallel to the $\xi$ axis and that in the outgoing beam under $45^{\circ}$ to the $\xi$ axis, we can retrieve the phase difference between the total field components

$$
\stackrel{\widetilde{r}}{=} 0, \xi \xi_{\xi}\left(\xi^{i, 0}, \eta^{i, 0}\right) a_{\mathcal{\xi}}^{i}\left(\xi^{i, 0}, \eta^{i, 0}\right)+\underline{\widetilde{r}}_{=1, \xi \xi}\left(\xi^{i, 1}, \eta^{i, 1}\right) a_{\mathcal{\xi}}^{i}\left(\xi^{i, 1}, \eta^{i, 1}\right),
$$

and

$$
\stackrel{\widetilde{r}}{=} 0, \eta \xi_{\xi}\left(\xi^{i, 0}, \eta^{i, 0}\right) a_{\xi}^{i}\left(\xi^{i, 0}, \eta^{i, 0}\right)+\stackrel{\widetilde{r}}{=1, \eta \xi}_{\xi}\left(\xi^{i, 1}, \eta^{i, 1}\right) a_{\xi}^{i}\left(\xi^{i, 1}, \eta^{i, 1}\right),
$$

since the complex incident field $\vec{a}^{i}$ is assumed known and the amplitudes and phase difference of ${\underset{\underline{r}}{\widetilde{r}}}_{0, \mu \xi}$ and ${\underset{\underline{r}}{\widetilde{r}}}_{1, \mu \xi}$ have already been determined for $\mu=\xi$, $\eta$, we can retrieve the phase differences of all four reflection coefficients.

By repeating this procedure for a linear polarizer parallel to the $\eta$ axis in the incident beam, we retrieve similarly the phase differences between ${\underset{\underline{r}}{\underline{r}}}_{0, \mu \eta}$ and ${\underset{\underline{r}}{=0, \mu \eta}}_{\text {for }} \mu=\xi, \eta$. Hence the phase differences between all elements of the reflection matrices $\underline{\underline{r}}_{0}$ and $\widetilde{\widetilde{r}}_{1}$ that are in different rows but the same column are retrieved.

Finally, we insert a polarizer in the reflected beam and subsequently use in the incident beam a polarizer at $45^{\circ}$ to obtain the phase differences between the elements of the two reflection matrices that are in different columns but the same row. We conclude that the complex reflection matrices ${\underset{\underline{\underline{r}}}{0}}_{0}$ and $\underline{\widetilde{r}}_{1}$ can be retrieved except for a common constant phase.

\subsection{Summary}

In summary, for points in the region of the pupil without overlapping of reflected orders, only the specular reflected field contributes. Hence only the reflection matrix $\underset{=}{\widetilde{r}}$ influences the measurements. By the procedure using linear polarizers and a half wave plate as described above, the complex elements of this reflection matrix are retrieved except for a common constant phase.

For points in the pupil for which several orders overlap, a number of reflection matrices $\underline{\underline{r}}_{j}, j=1, \ldots, M$ contribute. By using $2 M+1$ scan positions and using the procedure with the linear polarizers and the half wave plate in the incident and reflected beams, we can retrieve all contributing complex reflection matrices except for a common constant phase.

In case $F>2$, for all points of the pupil of the lens, only the specular reflected wave contributes. Hence when $F>2$ there is no region of overlap of reflected orders inside the pupil and scanning of the spot is superfluous.

\section{EXPERIMENT AND SIMULATIONS}

In this section we apply the theory derived in Section 2. The proof of principle of the phase retrieval between the scattered orders from the far field intensity data is shown. Additionally a simulation study is performed for polarization dependent phase sensitivity of the grating parameters.

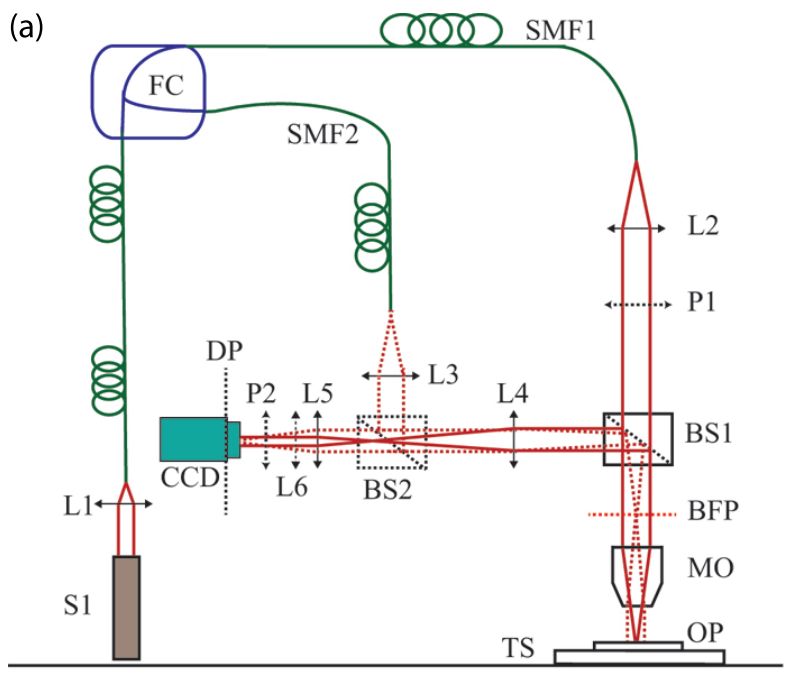

S1: He-Ne laser, FC: Fiber coupler, SMF: Single mode fiber, BS: Beam splitter, P: polarizer, L: lens, DP: Detector Plane, BFP: Back focal plane, OP: Object plane (grating),MO: Microscope objective, TS: Translation stage.
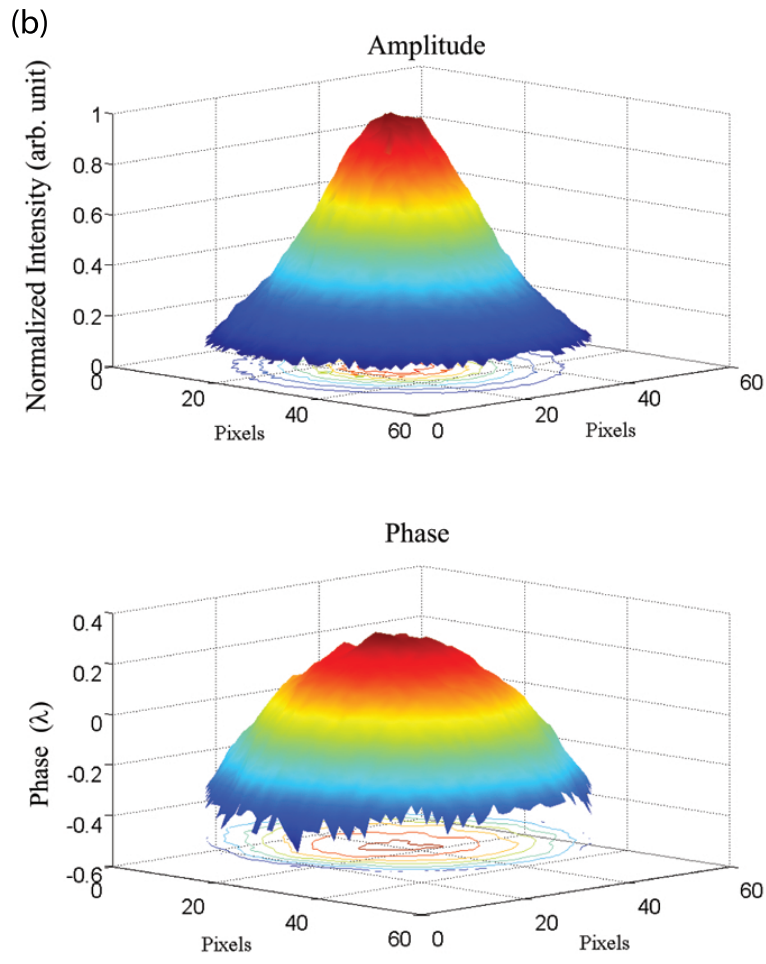

FIC. 2 (a) Schematic of the experimental set-up, (b) Measured normalized incident amplitude (top) and phase (bottom) in units of the wavelength of illumination above the microscope objective.

\subsection{Experimental setup}

Figure 2(a) shows the experimental setup. Linearly polarized light from a He-Ne laser (S1) is coupled to a fiber beam splitter (FC). The two output channels from the fiber coupler are indicated in the Figure 2 as SMF1 and SMF2. SMF1 is the illumination arm and SMF2 is the alignment arm in the setup. The polarizer (P1) selects the incident polarization of the collimated beam from SMF1. The polarized beam is then focused on the grating with a microscope objective $(\mathrm{MO})$ of numerical aperture 0.4 (Leica: N PLAN L 20x/0.40). The radius of the focused spot is $\approx 0.95 \mu \mathrm{m}$. The grating is placed on a piezo-controlled translation stage that is used to scan the bias positions. After interaction, the scattered light is collected by the same micro- 


\section{Experiment}

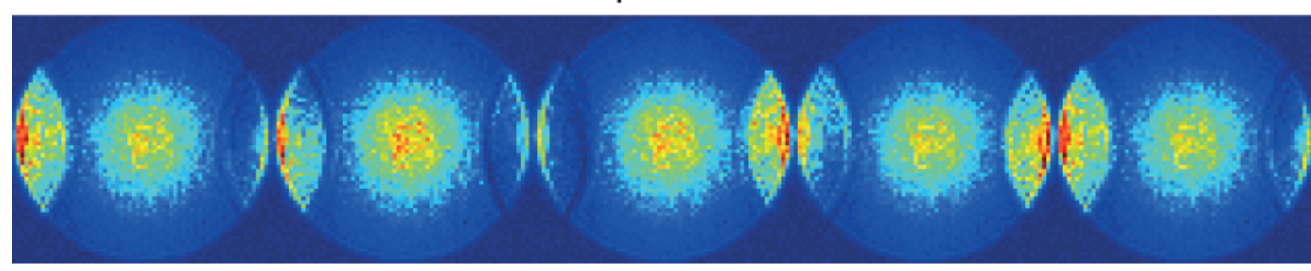

Simulation

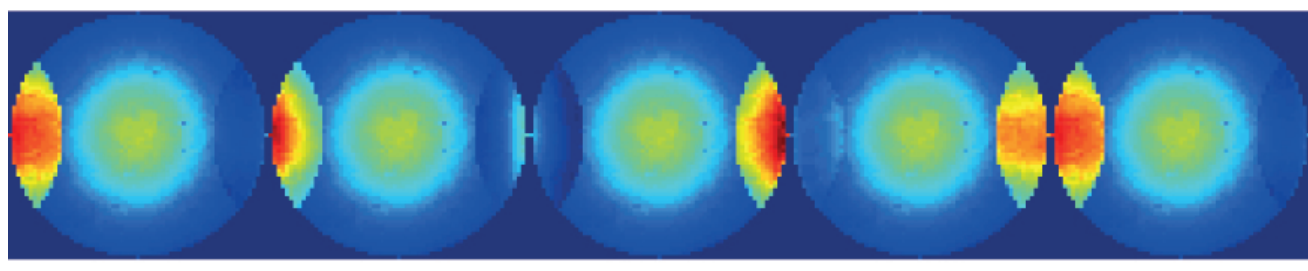

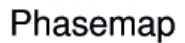

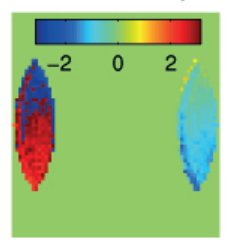

Phasemap

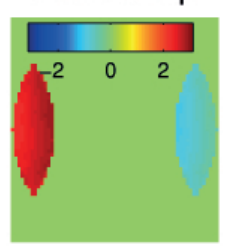

FIG. 3 Comparison between experiments and simulation result (for NA 0.4). The top row shows the experimental far fields corresponding to five scan positions within a single period of the grating and the phasemap computed from the far fields, in the bottom row is the corresponding simulated data.

\begin{tabular}{|l|c|}
\hline Grating Parameters & Nominal values \\
\hline \hline Period & $1 \mu \mathrm{m}$ \\
\hline MidCD & $425 \mathrm{~nm}$ \\
\hline Height & $155 \mathrm{~nm}$ \\
\hline SWA & $90^{\circ}$ \\
\hline
\end{tabular}

TABLE 1 Nominal grating parameters used in experiment and simulation.

scope objective. The far field is registered at the CCD plane after passing through the telescopic lens system L4 and L5. The telescopic system demagnifies the beam and also relays the far field at back focal plane of the MO to the CCD. The output polarization of the reflected wave is selected by polarizer (P2) placed before the CCD. A collimated beam from SMF2 is used for alignment of L4 of the telescopic system. Focal point of L4 is aligned to the back focal plane of the microscope objective. L5 is aligned by measuring the collimation after the telescopic system, when the collimated beam from SMF1 is focused on the plane of the $\mathrm{Si}$ wafer acting as the mirror. The present telescopic system is a demagnifier of $2.5 \mathrm{X}$. L6 can also be used for imaging the wafer with SMF2 illumination, and it is removed in the process of data acquistion. The grating parameters used in the simulations and experiment are given in Table 1. The far field intensity maps registered at the CCD are compared to the simulated data.

To compare theory and experiment we compute the reflected field with Rigorous Coupled Wave Analysis (RCWA). The amplitude and phase of the incident beam is measured with a Shack-Hartmann sensor as shown in Figure 2(b). Far field intensity maps for all polarizations are simulated using an input field of measured wavefront. The experimental results shown in Figure 3 are restricted to the case of the $\xi$ polarisation component of the reflected field for $\xi$ polarized input field.

In the top row of Figure 3, we show the measured far field intensity corresponding to the five scan positions within a single period of the grating and the phase map computed analytically from the far field intensity maps. In the bottom row the simulated data is shown. The separation between two adja- cent scanning positions is $\Lambda / 4$, hence the fifth and the first far field are the same. Based on simulation studies, it is observed that the phase map is robust to the noise of the system. We assume that the intensity is normally distributed with standard deviations given by $\sigma=2.7 \times 10^{-3}$ from the measured data. Experimental and the simulated far fields correspond to a fixed bias value of the grating.

As one can see, most features predicted in simulations are observed in the experiment. The phase map on the right side of the Figure 3 are obtained by the analysis given in Appendix A. We also see that the information content of the phase map is limited to the overlap regions of the diffracted orders as expected.

\subsection{Phase sensitivity to grating parameters}

Figure 4 summarizes the polarization dependent sensitivity of the grating parameters for the computed phase. We define the nominal values of grating parameters as given in Table 1 , and we look for the changes in the computed phase map for change in grating parameters from their nominal values. On the horizontal axis of Figure 4, one of the grating parameters are allowed to vary between $\pm 5 \%( \pm 10 \%$ in bias) from its nominal value while other parameters remain fixed at their nominal values. Phase map is computed for the nominal and non-nominal values of the grating parameters for all sets of input and output polarization of the incident and diffracted beam, respectively. The effect of change in parameter value is characterized by the corresponding peak to valley (PV) difference in phase maps of nominal and non-nominal parameters. This PV is shown on the vertical axis of Figure 4.

We observe that the grating parameters MidCD and height are most sensitive for $\xi_{\text {in }} \xi_{\text {out }}$ ( $\xi$ incident and $\xi$ output) polarization, while SWA sensitivity is maximum for crossed polarization namely $\eta_{\text {in }} \xi_{\text {out }}$. Position dependency i.e., bias shows identical sensitivity to all sets of incident and output polarizations, as expected. 

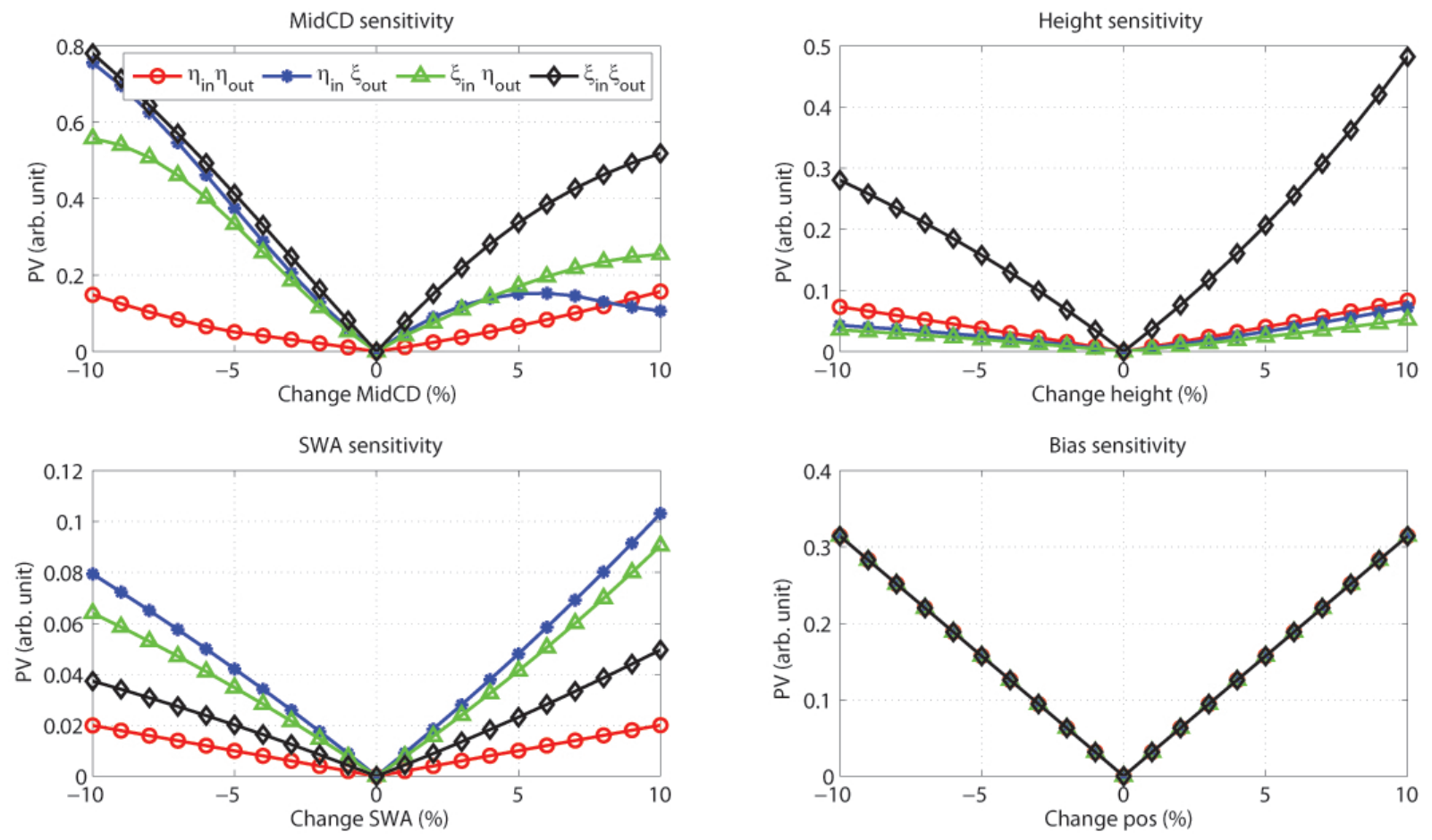

FIG. 4 Polarization dependent phase sensitivity for grating parameters MidCD, Height, SWA and Bias for different set of input and output polarizations. The legend for all the graphs are identical to the graph in top left of the figure.

\section{ANALYSIS AND CONCLUSIONS}

The distribution in phase map is dependent upon the geometrical parameters of the grating and the bias. The phase dependency on the geometrical parameters can be used in grating reconstruction in addition to the intensity data. The combined effect of intensity and phase map can be used to more robust grating reconstruction. Also the bias dependency on the phase map can be utilized for very accurate positioning of the gratings in for example, alignment, overlay metrology and nano-patterning. Intensity data with phase between scattered orders is the maximum information that can be extracted in CFS, as we have the amplitude and phase of all the four components of the scattering matrix except a constant phase. This constant phase can be retrieved with CFS in combination with, for example interferometry. The accuracy of the technique described in the present work mainly depends on the noise of the system which, in turn, accounts for the noise of the detector, quality of the optical components and mechanical stability. Other parameters which affect the sensitivity are the quality (and knowledge) of the polarization state of the incident light and the stability of piezo-controlled scanning stage. Finally, the method requires a proper characterization of the field in the entrance pupil of the system, both in terms of phase and amplitude for each of the two orthogonal polarization states. In case of a perfect optical system, then the ultimate sensitivity is exclusively determined by the noise level of the detector.

In conclusion, the applicability of Temporal Phase Shifting Interferometry in Coherent Fourier Scatterometry with a scanning spot is presented. An analytical relation is derived and illustrated for the phase difference between two overlapping orders in the exit pupil. An experimental setup was built to demonstrate the principle and the results were compared with rigourous simulations. The polarization dependent phase sensitivity of the grating parameters is presented. The importance of phase information in inverse problem of grating reconstruction is highlighted and the maximum information content of the CFS with a scanning spot is discussed.

\section{ACKNOWLEDGEMENTS}

N. Kumar and O. El Gawhary acknowledge the European FP7 Marie Curie research and initial training program, Surface Physics for Advanced Manufacturing for funding the research under the project number 215723. N. Kumar acknowledges Mark Van Kraaij from ASML for the RCWA code, A. Wiegmann (PTB, Germany) and Peter Petrik (TU Delft) for fruitful discussions. We also thank EMRP Joint Research Project IND 17 Scatterometry. The EMRP is jointly funded by the EMRP participating countries within EURAMET and the European Union.

\section{A BUCKET ALGORITHM FOR ANALYTICAL RECONSTRUCTION OF THE PHASE DIFFERENCE OF OVERLAPPING ORDERS}

Let $1<F<2$, and let the input and output polarizations be denoted by index $\mu$ and $v$, respectively, where $\mu=\xi, \eta ; v=$ $\xi, \eta$. Then for a translation $\Delta x$ of the grating, the total reflected field in a point of the exit pupil where the 0 and 1 th reflected 
orders overlap is given by:

$$
\begin{aligned}
& I_{\mu \nu}^{\Delta x}=\left|\widetilde{\underline{r}}_{\underline{=}, \mu \nu} \vec{a}_{\mu}^{i, 0}\right|^{2}+\left|\widetilde{\underline{r}}_{1, \mu \nu} \vec{a}_{\mu}^{i, 1}\right|^{2}
\end{aligned}
$$

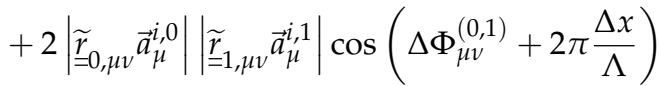

where the superscripts 0 and 1 of the incident field are to indicate that the incident field has to be evaluated in the pupil points corresponding to the $0^{\text {th }}$ and $1^{\text {st }}$ reflected orders. If the grating is scanned within one period such that $2 \pi \Delta x / \Lambda=\pi / 2, \pi, 3 \pi / 2$ and $2 \pi$, the measured intensities are

$$
\begin{aligned}
& I_{\mu \nu}^{x_{0}}=\left|\underline{\widetilde{r}}_{=0, \mu \nu} \vec{a}_{\mu}^{i, 0}\right|^{2}+\left|\underline{\widetilde{r}}_{1, \mu \nu} \vec{a}_{\mu}^{i, 1}\right|^{2} \\
& +2\left|\widetilde{\underline{r}}_{=0, \mu \nu} \vec{a}_{\mu}^{i, 0}\right||| \widetilde{r}_{1, \mu \nu} \vec{a}_{\mu}^{i, 1} \mid \cos \left(\Delta \Phi_{\mu \nu}^{(0,1)}\right) \\
& I_{\mu \nu}^{x_{1}}=\left|\underline{\underline{r}}_{=0, \mu \nu} \vec{a}_{\mu}^{i, 0}\right|^{2}+\left|\underline{\underline{r}}_{1, \mu \nu} \vec{a}_{\mu}^{i, 1}\right|^{2} \\
& -2\left|\widetilde{\underline{r}}_{=0, \mu \nu} \vec{a}_{\mu}^{i, 0}\right|\left|\tilde{\underline{r}}_{1, \mu \nu} \vec{a}_{\mu}^{i, 1}\right| \sin \left(\Delta \Phi_{\mu \nu}^{(0,1)}\right) \\
& I_{\mu \nu}^{x_{2}}=\left|\underline{\widetilde{r}}_{=0, \mu \nu} \vec{a}_{\mu}^{i, 0}\right|^{2}+\left|\underline{\underline{r}}_{1, \mu \nu} \vec{a}_{\mu}^{i, 1}\right|^{2} \\
& -2\left|\widetilde{\underline{r}}_{=0, \mu \nu} \vec{a}_{\mu}^{i, 0}\right||| \widetilde{r}_{1, \mu v} \vec{a}_{\mu}^{i, 1} \mid \cos \left(\Delta \Phi_{\mu \nu}^{(0,1)}\right) \\
& I_{\mu \nu}^{x_{3}}=\left|\widetilde{\underline{r}}_{=0, \mu \nu} \vec{a}_{\mu}^{i, 0}\right|^{2}+\left|\underline{\underline{r}}_{1, \mu \nu} \vec{a}_{\mu}^{i, 1}\right|^{2} \\
& +2\left|\widetilde{\underline{r}}_{=0, \mu \nu} \vec{a}_{\mu}^{i, 0}\right||| \widetilde{\underline{r}}_{1, \mu \nu} \vec{a}_{\mu}^{i, 1} \mid \sin \left(\Delta \Phi_{\mu \nu}^{(0,1)}\right)
\end{aligned}
$$

Rearranging the above equations, we can retrieve the phase difference of the contributing reflected orders for the chosen polarizations as:

$$
\Delta \Phi_{\mu \nu}^{(0,1)}=\arctan \frac{I_{\mu \nu}^{x_{3}}-I_{\mu \nu}^{x_{1}}}{I_{\mu \nu}^{x_{2}}-I_{\mu \nu}^{x_{0}}}
$$

\section{References}

[1] H. Gross, R. Model, M. Bar, M. Wurm, B. Bodermann, and A. Rathsfeld, "Mathematical modelling of indirect measurements in scatterometry," Measurement 39, 782-794 (2006).

[2] H. Gross, J. Richter, A. Rathfeld, and M. Bar, "Investigations on a robust profile model for the reconstruction of $2 \mathrm{D}$ periodic absorber lines in scatterometry," J. Europ. Opt. Soc. Rap. Public. 5, 10053 (2010).
[3] M. Wurm, F. Pilarski, and B. Bodermann, "A new flexible scatterometer for critical dimension metrology," Rev. Sci. Instrum. 81, 023701 (2010).

[4] R. M. Silver, B. M. Barnes, A. Heckert, R. Attota, R. Dixson, and J. Jun, "Angle resolved optical metrology," Proc. SPIE 6922, 69221M (2008).

[5] M. Wurm, S. Bonifer, B. Bodermann, and M. Gerhard, "Comparison of far field characterisation of DOEs with a goniometric DUVScatterometer and a CCD-based system," J. Europ. Opt. Soc. Rap. Public. 6, 11015s (2011).

[6] 0. El Gawhary, N. Kumar, S. F. Pereira, W. M. J. Coene, and H. P. Urbach, "Performance analysis of coherent optical scatterometry," Appl. Phys. B 105, 775-781 (2011).

[7] N. Kumar, 0. El Gawhary, S. Roy, V. G. Kutchoukov, S. F. Pereira, W. Coene, and H. P. Urbach, "Coherent Fourier scatterometry: tool for improved sensitivity in semiconductor metrology," Proc. SPIE 8324, 83240Q (2012).

[8] S. Roy, 0. El Gawhary, N. Kumar, S. F. Pereira, and H. P. Urbach, "Scanning effects in coherent fourier scatterometry," J. Europ. Opt. Soc. Rap. Public. 7, 12031 (2012).

[9] P. Hariharan, Basics of interferometry, second edition (Academic Press, Amsterdam, 2007).

[10] J. R. Fienup, "Phase retrieval algorithms: A comparison," Appl. Optics 21, 2758-2769 (1982).

[11] H. M. L. Faulkner and J. M. Rodenburg, "Movable aperture lensless transmission microscopy: A novel phase retrieval algorithm," Phys. Rev. Lett. 93, 023903-1 (2004).

[12] J. Miao, D. Sayre, and H. N. Chapman, "Phase retrieval from the magnitude of the Fourier transforms of nonperiodic objects," J. Opt. Soc. Am. A 15, 1662-1669 (1998).

[13] M. Born, and E. Wolf, Principles of Optics (Cambridge University Press, 1998).

[14] D. W. Robinson, G. T. Reid, and P. D. Groot, "Interferogram Analysis: Digital Fringe Pattern Measurement Techniques," Phys. Today 47, 66 (1994).

[15] R. R. Cordero, J. Molimard, A. Martínez, and F. Labbe, “Uncertainty analysis of temporal phase-stepping algorithms for interferometry," Opt. Commun. 275, 144-155 (2007).

[16] V. S. Ignatowsky, "Diffraction by lens of arbitrary aperture," Trans. Opt. Inst. Petrograd 1 (4), 1-36 (1919).

[17] B. Richards, and E. Wolf, "Electromagnetic diffraction in optical systems II. Structure of the image field in an aplanatic system," Proc. R. Soc. London Ser. A 253, 353-358 (1959).

[18] L. Novotny, and B. Hecht, Principles of Nano-Optics (Cambridge University Press, 2006). 